\title{
On the Probabilistic Characteristics of A Two Lane Slab-Type-Bridge Response Due to Traffic Flow
}

\author{
L. M. Anague Tabejieu ${ }^{1 *}$ and B. R. Nana Nbendjo ${ }^{1}$ \\ ${ }^{1}$ Laboratory of Modelling and Simulation in Engineering, Biomimetics and Prototypes, Faculty of Science, University of Yaoundé I, P.O. Box \\ 812, Yaoundé, Cameroon. \\ * Corresponding author
}

\author{
Article Info \\ Keywords: Bridge deflection, Thin rect- \\ angular plate, Traffic flow, Two lane \\ slab-type-bridge. \\ 2010 AMS: 00A69 \\ Received: 27 June 2018 \\ Accepted: 21 March 2019 \\ Available online: 30 August 2019
}

\begin{abstract}
In this study, an evaluation approach to obtain the two first probabilistic characteristics of a two lane slab-type-bridge response due to traffic flow is investigated. A two lane slab-type-bridge is modelled by a Simply Supported (SS) thin rectangular plate with two separate rectilinear paths. The modelling of the traffic flow is based on the assumption that two opposite series of vehicles of random weights arrive at the bridge at random times that constitute the Poisson stochastic process and with the stochastic velocities. The expected value and the standard deviation of the bridge deflection are obtained. We demonstrate that the bridge safety strongly depends to the mean value and to the standard deviation of the vehicles velocities.
\end{abstract}

\section{Introduction}

Vibration of a structure subjected to moving loads is of great theoretical and practical significance in civil engineering. Vibrations connected with this issue occur for example in the roadways loaded by traffic. [1] and [2] addressed a variety of engineering problems which are concerned with the dynamics of structures subjected to moving loads. Different types of structures and girders like beams, plates, shells, frames were considered and also different models of moving loads were assumed. For example, The dynamics response of the plate under moving load was considered by many authors but in most of their works the moving load was regarded as deterministic. A concise review of several related research studies is carried out in the following paragraph of this paper.

[3] proposed a classical closed-loop control algorithm to suppress the vibration of a simply-supported rectangular plate excited by a moving mass via a number of bonded active piezoelectric patches. [4] examined an inclined flat plate vibration due to traveling loads. [5] provided a semi-analytical simulation of a shear deformable plate vibration due to traveling inertial loads considering a general load distribution pattern and plate boundary condition. [6] analysed the dynamic behavior of an orthotropic plate simply supported on a pair of parallel edges and under a system of moving loads based on Lagrange equation and modal superposition. Preliminary results of the paper indicated that the multilane loading case is less critical than a single-lane loading case. [7] considered two series of moving inertial loads traversing the plate surface along parallel rectilinear trajectories with opposite directions and studied the resonance caused by this loads.

In real situation the load process has stochastic nature. So, the problem of vibrations of a plate subjected to this type of load was considered in some papers in stochastic approaches. [8] investigated the problem of a dynamic response of an infinite beam and a plate resting on a two-parametric foundation (Pasternak foundation) to the passage of a train of random forces. They developed an analytical technique to determine the two first probabilistic characteristics of the beam and plate responses. [9] investigated the dynamic response of a rectangular plate on a viscoelastic foundation under moving loads with varying velocity. The deflection distribution of the plate and the effects of the type of motion, initial speed of the load and foundation damping on the plate response are illustrated and analyzed.

In order to distinguish the present work from the above pertinent literature, we propose here to extend the work done by [7] by taking into account the more realistic model of moving loads (when it idealizes the vehicles on the bridge). In this model all the forces (vehicles) arrive at the plate at the random time instant, with stochastic velocities. So, a semi-analytical approach ([14]) to obtain the probabilistic characteristics of a two lane slab-type-bridge response due to two opposite series of stochastic moving loads is investigated. We call the attention of the reader about the fact that, the stochastic perturbation method proposed by [18] can be also used to achieve the same goal. 
Thus in this paper, the probabilistic characteristics of the plate response are sought in the form of the two first probabilistic moments, i.e. the expected value and the variance function.

The structure of this paper is as follows. After this Introduction in Section 1, the bridge model is presented and described in Section 2; the dynamic equations of motion for the vehicles-bridge are derived by using the Newton second law's; the Galerkin's method is used to discretize the equations. Section 3 presents a semi-analytical approach to obtain the probabilistic characteristics of the bridge plate response and some numerical results. Finally, conclusions are drawn in section 4.

\section{The Bridge model and the mathematical formulation}

Let us consider a model of a two lane slab-type-bridge (see Fig. 2.1(a)) modelled by a SS thin rectangular plate (of length $a$, width $b$ and thickness $h$ ) with two separate rectilinear paths and subjected to two opposite series of moving forces with random values $A_{i j}$ (vehicle weights) appearing at random times $t_{i j}$, as shown in Fig. 2.1(b). Such an excitation process is an appropriate model of vehicular traffic loads

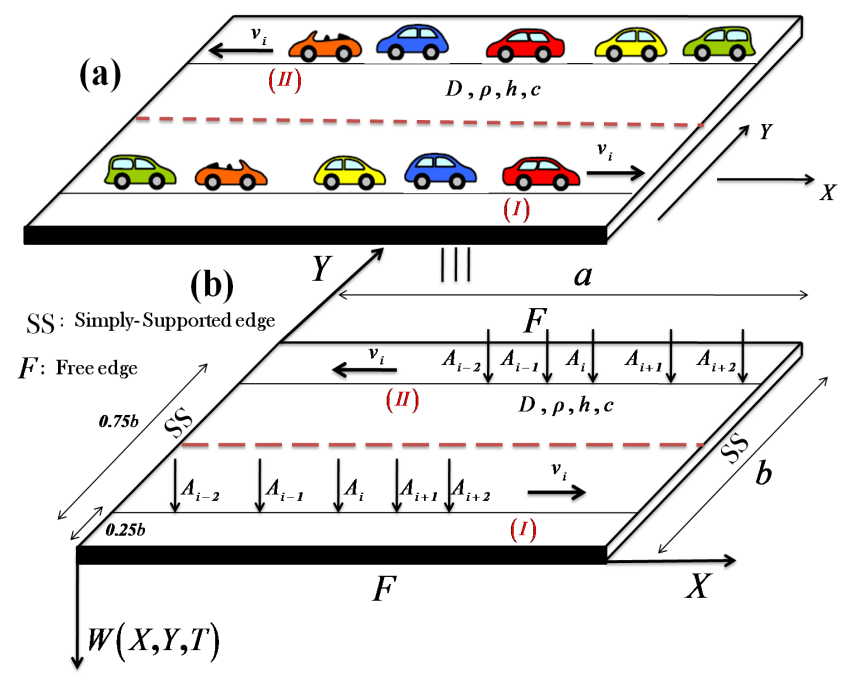

Figure 2.1: The general plan of the model of a two lane slab-type bridge and its loading (a). The simplified scheme of the model (b).

acting on the bridge. The plate is assumed to behave according to the Kirchhoff's hypothesis [10]. Thus, the vibrations of the plate due to these random forces are described by the following equation:

$$
\rho h \frac{\partial^{2} W}{\partial T^{2}}+c \frac{\partial W}{\partial T}+D\left[\frac{\partial^{4} W}{\partial X^{4}}+\frac{\partial^{4} W}{\partial X^{2} \partial Y^{2}}+\frac{\partial^{4} W}{\partial Y^{4}}\right]=P(X, Y, T)
$$

where $W(X, Y, T)$ denotes vertical deflection of the plate at point $X, Y$, and time $T . D=E h^{3} / 12\left(1-v^{2}\right)$ is the bending rigidity of the plate, with $h, E, v$ are the thickness, elastic modulus and Poisson's ratio of the plate, respectively. $\rho$ represents the mass per unit of volume of the plate, $c$ the damping coefficient of the plate and $P(X, Y, T)$ denotes the load process. In the case of a random train of forces (vehicles) moving in opposite directions in a two separate rectilinear paths $\left(n_{l}=2\right)$, the loading process has a form:

$$
P(X, Y, T)=\sum_{j=1}^{n_{l}} \sum_{i=1}^{N_{j}(T)} A_{i j} \delta\left[X-X_{i j}\left(T-T_{i j}\right)\right] \delta\left[Y-Y_{0 j}\right]
$$

where $\delta[\ldots]$ stands for the Dirac delta function, $A_{i j}$ are the weights which are assumed to be random variables, mutually independent and also independent of the instants $t_{i j}$ (arrival times of the moving forces), $Y_{0 j}$ is the deterministic y-coordinate that fixes the position of each traffic lane, $n_{l}$ is the number of traffic lane considered and $N_{j}(T)$ is the number of forces that acted on the plate from the beginning of observation up to the time $T$ and that describes the Poisson process. $X_{i j}\left(T-T_{i j}\right)$ is the distance covered by the $i-t h$ force moving one by one to the time $T$ on the $j-t h$ traffic lane. It is assumed that one of a random train of forces traverses the plate on the following rectilinear trajectory $([14,12])$ :

$$
\begin{aligned}
& \frac{d X_{i j}\left(T-T_{i j}\right)}{d T}=v_{i j}\left(T-T_{i j}\right)=\varepsilon_{j} v_{0 j}+\sigma_{v j} \xi_{i j}\left(T-T_{i j}\right) \\
& Y_{0 j}=\left\{\begin{array}{cl}
0.25 b & \text { For the trajectory }(\mathrm{I}), \varepsilon_{j}=1 \\
0.75 b & \text { For the trajectory (II) }, \varepsilon_{j}=-1
\end{array}\right. \\
& 0 \leq X_{i j}\left(T-T_{i j}\right) \leq a
\end{aligned}
$$

where $v_{i j}\left(T-T_{i j}\right)$ is the stochastic velocity of the $i-t h$ force, that moving in the traffic lane $j . v_{0 j}$ presents the mean value of velocity, $\sigma_{v j}$ its standard deviation and $\xi_{i j}\left(T-T_{i j}\right)$ the velocity disturbance. The function $\varepsilon_{j}$ is introduced and defined as: $\varepsilon_{j}=1$ when the loads are crossing the plate in one of their rectilinear path ( situation (I) of Fig. 2.1), and $\varepsilon_{j}=-1$ for the other line of the loads trajectory (situation (II) 
of Fig. 2.1). It is assumed that, the force disturbances $\xi_{i j}\left(T-T_{i j}\right)$ are stationary random processes, here the mutually independent Gaussian white noise processes, i.e.

$$
\begin{aligned}
& \mathrm{E}\left[v_{i j}\left(T-T_{i j}\right)\right]=v_{0 j}, \mathrm{E}\left[\xi_{i j}\left(T-T_{i j}\right)\right]=0 \\
& \mathrm{E}\left[\xi_{i j}\left(T-T_{i j}\right) \xi_{k l}\left(T-T_{i j}\right)\right]=0 \text { for }(k, l) \neq(i, j), \\
& \mathrm{E}\left[\xi_{i j}\left(T-T_{i j}\right) \xi_{i j}\left(T-T_{i j}+\varsigma\right)\right]=\Delta_{j}^{2} \delta(\varsigma)
\end{aligned}
$$

where $\mathrm{E}[\ldots]$ denotes the expected value of the quantity in brackets and $\Delta_{j}=v_{o j} \sigma_{v j}$.

In order to improve the accuracy of the numerical calculation, the following dimensionless variables are defined as follows:

$$
w=\frac{W}{h}, x=\frac{X}{a}, y=\frac{Y}{b}, t=\omega_{0} T, x \in[0,1], y \in[0,1]
$$

Eq. (2.1) takes the form:

$$
\frac{\partial^{2} w}{\partial t^{2}}+\mu \frac{\partial w}{\partial t}+\frac{\partial^{4} w}{\partial x^{4}}+2\left(\frac{a}{b}\right)^{2} \frac{\partial^{4} w}{\partial x^{2} \partial y^{2}}+\left(\frac{a}{b}\right)^{4} \frac{\partial^{4} w}{\partial y^{4}}=P(x, y, t)
$$

with

$$
\omega_{0}=\frac{1}{a^{2}} \sqrt{\frac{D}{\rho h}}, \mu=\frac{c a^{2}}{\sqrt{\rho h D}}, P(x, y, t)=\frac{a^{4}}{D h} P(X, Y, T)
$$

Eq. (2.3) takes the dimensionless form:

$$
\begin{aligned}
& \frac{d x_{i j}\left(t-t_{i j}\right)}{d t}=u_{i j}\left(t-t_{i j}\right)=\varepsilon_{j} u_{0 j}+\sigma_{v j} \zeta_{i j}\left(t-t_{i j}\right) \\
& y_{0 j}=\left\{\begin{array}{c}
0.25 \text { For the trajectory }(\mathrm{I}), \varepsilon_{j}=1 \\
0.75 \text { For the trajectory (II), } \varepsilon_{j}=-1
\end{array}\right. \\
& 0 \leq x_{i j}\left(t-t_{i j}\right) \leq 1
\end{aligned}
$$

where:

$$
u_{0 j}=\frac{v_{0 j}}{a \omega_{0}}, \quad \zeta_{i j}=\frac{\xi_{i j}}{a \omega_{0}}, x_{i j}=\frac{X_{i j}}{a}, y_{0 j}=\frac{Y_{0 j}}{b} .
$$

Since the parameters of the plate are deterministic, let the dynamic influence function $H_{j}\left(x, y, t-t_{i j}\right)$ denote the response of the plate at the time $t$ to the moving force when the amplitude $A_{i j}=1$. This function satisfies the following equation:

$$
\frac{\partial^{2} H_{j}}{\partial t^{2}}+\mu \frac{\partial H_{j}}{\partial t}+\frac{\partial^{4} H_{j}}{\partial x^{4}}+2\left(\frac{a}{b}\right)^{2} \frac{\partial^{4} H_{j}}{\partial x^{2} \partial y^{2}}+\left(\frac{a}{b}\right)^{4} \frac{\partial^{4} H_{j}}{\partial y^{4}}=\frac{a^{3}}{D h b} 1 . \delta\left[x-x_{i j}\left(t-t_{i j}\right)\right] \delta\left[y-y_{0 j}\right]
$$

and the following boundary (Simply supported one) and initial conditions are considered

$$
\begin{aligned}
& \left.H_{j}\left(x, y, t-t_{i j}\right)\right|_{x=0}=\left.H_{j}\left(x, y, t-t_{i j}\right)\right|_{x=1}=0 \\
& \left.H_{j}\left(x, y, t-t_{i j}\right)\right|_{y=0}=\left.H_{j}\left(x, y, t-t_{i j}\right)\right|_{y=1}=0, \\
& \left.\frac{\partial^{2} H_{j}\left(x, y, t-t_{i j}\right)}{\partial x^{2}}\right|_{x=0}=\left.\frac{\partial^{2} H_{j}\left(x, y, t-t_{i j}\right)}{\partial x^{2}}\right|_{x=1}=0, \\
& \left.\frac{\partial^{2} H_{j}\left(x, y, t-t_{i j}\right)}{\partial x^{2}}\right|_{y=0}=\left.\frac{\partial^{2} H_{j}\left(x, y, t-t_{i j}\right)}{\partial x^{2}}\right|_{y=1}=0, \\
& \left.H_{j}\left(x, y, t-t_{i j}\right)\right|_{t=t_{i j}}=0,\left.\frac{\partial H_{j}\left(x, y, t-t_{i j}\right)}{\partial t}\right|_{t=t_{i j}}=0 .
\end{aligned}
$$

To investigate the probabilistic response of the system let us derive the modal equations. To do so, Galerkin's method is applied. According to this method and by taking into account the boundary conditions of the plate, the solution of the partial differential Eq. (2.10) is assumed to be in the form:

$$
H_{j}\left(x, y, t-t_{i j}\right)=\sum_{n=1}^{\infty} \sum_{m=1}^{\infty} \chi_{n, m}^{(j)}\left(t-t_{i j}\right) \sin (n \pi x) \sin (m \pi y)
$$

where $\chi_{n, m}^{(j)}\left(t-t_{i j}\right)$ is the generalized coordinates, $\sin (n \pi x) \sin (m \pi y)$ is the dimensionless solution of the eigenvalue problem which depends on the boundary conditions of the free oscillations of the plate and $(n, m)$ is the natural mode with $n$ and $m$ nodal lines lying the $x-$ and $y$-directions, respectively. To apply the method, Eq. (2.12) is inserted into Eq. (2.10) and the resultant equation is multiplied by the corresponding eigenfunction and then integrated over the surface area of the plate. Thus, the dimensionless modal equation is obtained as:

where:

$$
\ddot{\chi}_{n, m}^{(j)}\left(t-t_{i j}\right)+\mu \dot{\chi}_{n, m}^{(j)}\left(t-t_{i j}\right)+\omega_{n m}^{2} \chi_{n, m}^{(j)}\left(t-t_{i j}\right)=\Gamma_{m}^{(j)} \sin \left[n \pi x_{i j}\left(t-t_{i j}\right)\right]
$$

$$
\Gamma_{m}^{(j)}=1 . \frac{4 a^{3} \sin \left(m \pi y_{0 j}\right)}{D h b}, \quad \omega_{n m}^{2}=\left[(n \pi)^{2}+\left(\frac{m \pi a}{b}\right)^{2}\right]^{2}
$$




\section{Stochastic analysis}

In this Section, an analytical approach for obtaining the probabilistic characteristics of the bridge plate response is developed (Subsect. 3.1) and some numerical results (Subsect. 3.2) are shown.

\subsection{Effective solution of the problem}

In order to directly evaluate the probabilistic characteristics of the system response under random train of moving loads, Eq. (2.13) needs to be transformed using the Itô integral and the Itô differentiation rule( [13], [14]). By introducing the following state variables,

$$
\begin{aligned}
& z_{1}^{(j)}(n, m, t)=\chi_{n, m}^{(j)}(t), z_{2}^{(j)}(n, m, t)=\dot{\chi}_{n, m}^{(j)}(t), \\
& z_{3}^{(j)}(n, m, t)=\sin \left[n \pi x_{i j}(t)\right], z_{4}^{(j)}(n, m, t)=\cos \left[n \pi x_{i j}(t)\right] .
\end{aligned}
$$

In which for the simplicity $t-t_{i j}$ is replaced by $t$. In view of Eq. (2.13) and according to the Itô Lemma [13], the corresponding stochastic problem for the state variables $z_{i}^{(j)} ;(i=1,2,3,4)$ may be written as a following set of Itô equations:

$$
\begin{aligned}
& \mathrm{d} z_{1}^{(j)}(n, m, t)=z_{2}^{(j)}(n, m, t) \mathrm{d} t \\
& \mathrm{~d} z_{2}^{(j)}(n, m, t)=\left[-\omega_{n m}^{2} z_{1}^{(j)}(n, m, t)-\mu z_{2}^{(j)}(n, m, t)+\Gamma_{m}^{(j)} z_{3}^{(j)}(n, m, t)\right] \mathrm{d} t \\
& \mathrm{~d} z_{3}^{(j)}(n, m, t)=n \pi \sigma_{v j} z_{4}^{(j)}(n, m, t) \mathrm{d} W_{i j}(t)+\left[n \pi \varepsilon_{j} u_{0 j} z_{4}^{(j)}(n, m, t)-0.5\left(n \pi \sigma_{v j}\right)^{2} z_{3}^{(j)}(n, m, t)\right] \mathrm{d} t \\
& \mathrm{~d} z_{4}^{(j)}(n, m, t)=n \pi \sigma_{v j} z_{3}^{(j)}(n, m, t) \mathrm{d} W_{i j}(t)+\left[-n \pi \varepsilon_{j} u_{0 j} z_{3}^{(j)}(n, m, t)-0.5\left(n \pi \sigma_{v j}\right)^{2} z_{4}^{(j)}(n, m, t)\right] \mathrm{d} t
\end{aligned}
$$

where $W_{i j}(t)$ is a unit Wiener stochastic process. By appropriately applying the mathematical expectation operator, the deterministic equations for various orders of the response moments can be derived. Thus, the dynamic response of the first order probabilistic moment is described by the following set of differential equations

$$
\begin{aligned}
& \frac{\mathrm{d} m_{1}^{(j)}(n, m, t)}{\mathrm{d} t}=m_{2}^{(j)}(n, m, t) \\
& \frac{\mathrm{d} m_{2}^{(j)}(n, m, t)}{\mathrm{d} t}=-\omega_{n m}^{2} m_{1}^{(j)}(n, m, t)-\mu m_{2}^{(j)}(n, m, t)+\Gamma_{m}^{(j)} m_{3}^{(j)}(n, m, t) \\
& \frac{\mathrm{d} m_{3}^{(j)}(n, m, t)}{\mathrm{d} t}=n \pi \varepsilon_{j} u_{0 j} m_{4}^{(j)}(n, m, t)-0.5\left(n \pi \sigma_{v j}\right)^{2} m_{3}^{(j)}(n, m, t) \\
& \frac{\mathrm{d} m_{4}^{(j)}(n, m, t)}{\mathrm{d} t}=-n \pi \varepsilon_{j} u_{0 j} m_{3}^{(j)}(n, m, t)-0.5\left(n \pi \sigma_{v j}\right)^{2} m_{4}^{(j)}(n, m, t)
\end{aligned}
$$

where $m_{i}^{(j)}(n, m, t)=\mathrm{E}\left[z_{i}^{(j)}(n, m, t)\right]$ and the initial conditions (according to Eqs. (2.11), (2.12) and (3.1)) are: $m_{1}^{(j)}(n, m, 0)=0$, $m_{2}^{(j)}(n, m, 0)=0, m_{3}^{(j)}(n, m, 0)=0, m_{4}^{(j)}(n, m, 0)=1$. For the second order dynamic moment, let us introduce the following notations for the second order probabilistic moments of variables $z_{i}^{(j)}(n, m, t)$

$$
\begin{aligned}
& m_{2000}^{(j)}(k, l, n, m, t)=\mathrm{E}\left[z_{1}^{(j)}(k, l, t) z_{1}^{(j)}(n, m, t)\right] \\
& m_{1100}^{(j)}(k, l, n, m, t)=\mathrm{E}\left[z_{1}^{(j)}(k, l, t) z_{2}^{(j)}(n, m, t)\right] \\
& m_{1010}^{(j)}(k, l, n, m, t)=\mathrm{E}\left[z_{1}^{(j)}(k, l, t) z_{3}^{(j)}(n, m, t)\right] \\
& m_{1001}^{(j)}(k, l, n, m, t)=\mathrm{E}\left[z_{1}^{(j)}(k, l, t) z_{4}^{(j)}(n, m, t)\right] \\
& m_{0200}^{(j)}(k, l, n, m, t)=\mathrm{E}\left[z_{2}^{(j)}(k, l, t) z_{2}^{(j)}(n, m, t)\right] \\
& m_{0110}^{(j)}(k, l, n, m, t)=\mathrm{E}\left[z_{2}^{(j)}(k, l, t) z_{3}^{(j)}(n, m, t)\right] \\
& m_{0101}^{(j)}(k, l, n, m, t)=\mathrm{E}\left[z_{2}^{(j)}(k, l, t) z_{4}^{(j)}(n, m, t)\right] \\
& m_{0020}^{(j)}(k, l, n, m, t)=\mathrm{E}\left[z_{3}^{(j)}(k, l, t) z_{3}^{(j)}(n, m, t)\right] \\
& m_{0011}^{(j)}(k, l, n, m, t)=\mathrm{E}\left[z_{3}^{(j)}(k, l, t) z_{4}^{(j)}(n, m, t)\right] \\
& m_{0002}^{(j)}(k, l, n, m, t)=\mathrm{E}\left[z_{4}^{(j)}(k, l, t) z_{4}^{(j)}(n, m, t)\right]
\end{aligned}
$$


So, the differential equations for calculating the second order probabilistic moments of the variables $z_{i}^{(j)}$ are obtained as:

$$
\begin{aligned}
\frac{\mathrm{d} m_{2000}^{(j)}(k, l, n, m, t)}{\mathrm{d} t}= & m_{1100}^{(j)}(k, l, n, m, t)+m_{1100}^{(j)}(n, m, k, l, t) \\
\frac{\mathrm{d} m_{1100}^{(j)}(k, l, n, m, t)}{\mathrm{d} t}= & m_{0200}^{(j)}(k, l, n, m, t)-\omega_{n m^{2}}^{2} m_{2000}^{(j)}(k, l, n, m, t)-\mu m_{1100}^{(j)}(k, l, n, m, t)+\Gamma_{m}^{(j)} m_{1010}^{(j)}(k, l, n, m, t) \\
\frac{\mathrm{d} m_{1010}^{(j)}(k, l, n, m, t)}{\mathrm{d} t}= & m_{0110}^{(j)}(k, l, n, m, t)+n \pi \varepsilon_{j} u_{0 j} m_{1001}^{(j)}(k, l, n, m, t)-0.5\left(n \pi \sigma_{v j}\right)^{2} m_{1010}^{(j)}(k, l, n, m, t) \\
\frac{\mathrm{d} m_{1001}^{(j)}(k, l, n, m, t)}{\mathrm{d} t}= & m_{0101}^{(j)}(k, l, n, m, t)-n \pi \varepsilon_{j} u_{0 j} m_{1010}^{(j)}(k, l, n, m, t)-0.5\left(n \pi \sigma_{v j}\right)^{2} m_{1001}^{(j)}(k, l, n, m, t) \\
\frac{\mathrm{d} m_{0200}^{(j)}(k, l, n, m, t)}{\mathrm{d} t}= & -\omega_{k l}^{2} m_{1100}^{(j)}(k, l, n, m, t)-\omega_{n m}^{2} m_{1100}^{(j)}(n, m, k, l, t)-2 \mu m_{0200}^{(j)}(k, l, n, m, t)+\Gamma_{m}^{(j)} m_{0110}^{(j)}(k, l, n, m, t)+\Gamma_{l}^{(j)} m_{0110}^{(j)}(n, m, k, l, t) \\
\frac{\mathrm{d} m_{0110}^{(j)}(k, l, n, m, t)}{\mathrm{d} t}= & -\omega_{k l}^{2} m_{1010}^{(j)}(k, l, n, m, t)-\mu m_{0110}^{(j)}(k, l, n, m, t)+\Gamma_{l}^{(j)} m_{0020}^{(j)}(k, l, n, m, t)+n \pi \varepsilon_{j} u_{0 j} m_{0101}^{(j)}(k, l, n, m, t) \\
& -0.5\left(n \pi \sigma_{v j}\right)^{2} m_{0110}^{(j)}(k, l, n, m, t) \\
\frac{\mathrm{d} m_{0101}^{(j)}(k, l, n, m, t)}{\mathrm{d} t}= & \left.-\omega_{k l}^{2} m_{1001}^{(j)}(k, l, n, m, t)-\mu m_{0101}^{(j)}(k, l, n, m, t)+\Gamma_{l}^{(j)} m_{0011}^{(j)}(k, l, n, m, t)-n \pi \varepsilon_{j} u_{0 j} m_{0110}^{(j)}(k, l, n, m, t)\right) \\
& -0.5\left(n \pi \sigma_{v j}\right)^{2} m_{0101}^{(j)}(k, l, n, m, t) \\
\frac{\mathrm{d} m_{0020}^{(j)}(k, l, n, m, t)}{\mathrm{d} t}= & \pi \varepsilon_{j} u_{0 j}\left[n m_{0011}^{(j)}(k, l, n, m, t)+k m_{0011}^{(j)}(n, m, k, l, t)\right]-0.5 \pi^{2} \sigma_{v j}^{2}\left[k^{2}+n^{2}\right] m_{0020}^{(j)}(k, l, n, m, t)+n k \pi^{2} \sigma_{v j}^{2} m_{0002}^{(j)}(k, l, n, m, t) \\
\frac{\mathrm{d} m_{0011}^{(j)}(k, l, n, m, t)}{\mathrm{d} t}= & k \pi \varepsilon_{j} u_{0 j} m_{0002}^{(j)}(k, l, n, m, t)-n \pi \varepsilon_{j} u_{0 j} m_{0020}^{(j)}(k, l, n, m, t)-0.5 \pi^{2} \sigma_{v j}^{2}\left[k^{2}+n^{2}\right] m_{0011}^{(j)}(k, l, n, m, t)+n k \pi^{2} \sigma_{v j}^{2} m_{0011}^{(j)}(k, l, n, m, t) \\
\frac{\mathrm{d} m_{0002}^{(j)}(k, l, n, m, t)}{\mathrm{d} t}= & -\pi \varepsilon_{j} u_{0 j}\left[k m_{0011}^{(j)}(k, l, n, m, t)+n m_{0011}^{(j)}(n, m, k, l, t)\right]-0.5 \pi^{2} \sigma_{v j}^{2}\left[k^{2}+n^{2}\right] m_{0002}^{(j)}(k, l, n, m, t)+n k \pi^{2} \sigma_{v j}^{2} m_{0020}^{(j)}(k, l, n, m, t) \\
&
\end{aligned}
$$

For $(k, l) \neq(n, m)$, the above set of differential equations must be completed by adding a duplicate set of equations in which the parameters $(k, l)$ should be replaced by $(n, m)$ and vice versa. According to Eqs. ((2.11), (2.12), (3.1) and (3.3)), the initial conditions are:

$$
\begin{aligned}
& m_{2000}^{(j)}(k, l, n, m, 0)=m_{2000}^{(j)}(n, m, k, l, 0)=0 \\
& m_{0200}^{(j)}(k, l, n, m, 0)=m_{0200}^{(j)}(n, m, k, l, 0)=0 \\
& m_{0020}^{(j)}(k, l, n, m, 0)=m_{0020}^{(j)}(n, m, k, l, 0)=0 \\
& m_{0002}^{(j)}(k, l, n, m, 0)=m_{0002}^{(j)}(n, m, k, l, 0)=1 \\
& m_{1100}^{(j)}(k, l, n, m, 0)=m_{1100}^{(j)}(n, m, k, l, 0)=0 \\
& m_{1010}^{(j)}(k, l, n, m, 0)=m_{1010}^{(j)}(n, m, k, l, 0)=0 \\
& m_{1001}^{(j)}(k, l, n, m, 0)=m_{1001}^{(j)}(n, m, k, l, 0)=0 \\
& m_{0110}^{(j)}(k, l, n, m, 0)=m_{0110}^{(j)}(n, m, k, l, 0)=0 \\
& m_{0101}^{(j)}(k, l, n, m, 0)=m_{0101}^{(j)}(n, m, k, l, 0)=0 \\
& m_{0011}^{(j)}(k, l, n, m, 0)=m_{0011}^{(j)}(n, m, k, l, 0)=0
\end{aligned}
$$

For the loading process, the solution of Eq. (2.6) is a superposition of individual response due to each train of forces moving to each lane. So the plate response may be expressed in the following form:

$$
w(x, y, t)=\frac{a^{3}}{D h b} \sum_{j=1}^{n_{l}} \sum_{i=1}^{N_{j}(t)} A_{i j} H_{j}\left(x, y, t-t_{i j}\right)=\frac{a^{3}}{D h b} \sum_{j=1}^{n_{l}} \int_{0}^{t} A_{j}(\tau) H_{j}(x, y, t-\tau) \mathrm{d} N_{j}(\tau)
$$

By taking into account the features of the Poisson process, we are looking for the expected value of the plate response in the form:

$$
\begin{aligned}
& \mathrm{E}[w(x, y, t)]=\frac{a^{3}}{D h b} \sum_{j=1}^{n_{l}} \mathrm{E}\left[A_{j}\right] \int_{0}^{t} \mathrm{E}\left[H_{j}\left(x, y, t-\tau_{j}\right)\right] \times \lambda_{j}(\tau) \wp\left\{x_{j}\left(t-\tau_{j}\right)<1\right\} \mathrm{d} \tau_{j} \\
& =\frac{a^{3}}{D h b} \sum_{j=1}^{n_{l}} \mathrm{E}\left[A_{j}\right] \sum_{n=1}^{\infty} \sum_{m=1}^{\infty} \sin (n \pi x) \sin (m \pi y) \times \int_{0}^{t} m_{1}^{(j)}\left(n, m, t-\tau_{j}\right) \lambda_{j}(\tau) \wp\left\{x_{j}\left(t-\tau_{j}\right)<1\right\} \mathrm{d} \tau_{j}
\end{aligned}
$$


and for the variance of the plate response in the form:

$$
\begin{aligned}
& \sigma_{w}^{2}(x, y, t)=\left(\frac{a^{3}}{D h b}\right)^{2} \sum_{j=1}^{n_{l}} \mathrm{E}\left[A_{j}^{2}\right] \int_{0}^{t} \mathrm{E}\left[H_{j}^{2}\left(x, y, t-\tau_{j}\right)\right] \times \lambda_{j}(\tau) \wp\left\{x_{j}\left(t-\tau_{j}\right)<1\right\} \mathrm{d} \tau_{j} \\
& =\sum_{j=1}^{n_{l}} \mathrm{E}\left[A_{j}^{2}\right] \sum_{k=1}^{\infty} \sum_{l=1}^{\infty} \sum_{n=1}^{\infty} \sum_{m=1}^{\infty} \sin (k \pi x) \sin (l \pi y) \sin (n \pi x) \sin (m \pi y) \times\left(\frac{a^{3}}{D h b}\right)^{2} \int_{0}^{t} m_{2000}^{(j)}\left(k, l, n, m, t-\tau_{j}\right) \lambda_{j}(\tau) \wp\left\{x_{j}\left(t-\tau_{j}\right)<1\right\} \mathrm{d} \tau_{j}
\end{aligned}
$$

where $\lambda_{j}(\tau)$ is the arrival rate for the $j-t h$ loads trajectory, $\wp\left\{x_{j}\left(t-\tau_{j}\right)<1\right\}$ is the unknown function which describing the fact that the probability that the forces occurring in time $\tau_{j}$ at the beginning of each rectilinear path $j$ of the plate is still acting on to the structure in time $t$. This function can be evaluated by first looking for the transition probability density function $f\left(x_{i j}, t-t_{i j}\right)$ for the process $x_{i j}\left(t-t_{i j}\right)$ (describes by Eq. (2.8) ) which is governed by the following Fokker-Planck-Kolmogorov equation.

$$
\frac{\partial f\left(x_{i j}, t-t_{i j}\right)}{\partial t}+\varepsilon_{j} u_{0 j} \frac{\partial f\left(x_{i j}, t-t_{i j}\right)}{\partial x_{i j}}+\frac{1}{2} \sigma_{v j}^{2} \frac{\partial^{2} f\left(x_{i j}, t-t_{i j}\right)}{\partial x_{i j}^{2}}=0
$$

By using the Fourier transform, the normalization condition and the following formulas,

$$
\left.f\left(x_{i j}, t-t_{i j}\right)\right|_{t=t_{i j}}=\delta\left(x_{i j}\right),\left.f\left(x_{i j}, t-t_{i j}\right)\right|_{x_{i j}=\infty}=0
$$

the solution of Eq. (3.10) is given as:

$$
\begin{aligned}
& f\left(x_{i j}, t-t_{i j}\right)=\frac{1}{\mu_{i j}} \sqrt{\frac{2}{\pi\left(t-t_{i j}\right)}} \exp \left[-\frac{\left(x_{i j}-\varepsilon_{j} u_{0 j}\left(t-t_{i j}\right)\right)^{2}}{2 \sigma_{v j}^{2}\left(t-t_{i j}\right)}\right] \\
& \text { For } x_{i j} \geq 0, j=1,2 .
\end{aligned}
$$

Thus, the probability $\wp\left\{x_{i j}\left(t-t_{i j}\right)<1\right\}$ that we are looking for takes a form:

$$
\wp\left\{x_{i j}\left(t-t_{i j}\right)<1\right\}=\int_{0}^{1} f\left(x_{i j}, t-t_{i j}\right) \mathrm{d} x_{i j}=\frac{1}{\mu_{i j} \sqrt{2}}\left[\sigma_{v j} \operatorname{Erf}\left(\sqrt{\frac{1}{2 \sigma_{v j}^{2}\left(t-t_{i j}\right)}}-\varepsilon_{j} \frac{u_{0 j}}{\sigma_{v j}} \sqrt{\frac{\left(t-t_{i j}\right)}{2}}\right)+\mu_{i j}-\sigma_{v j}\right]
$$

In which

$$
\mu_{i j}=\sigma_{v j}\left[1-\operatorname{Erf}\left(-\varepsilon_{j} \frac{u_{0 j}}{\sigma_{v j}} \sqrt{\frac{\left(t-t_{i j}\right)}{2}}\right)\right]
$$

$\operatorname{Erf}(\ldots)$ is the error function.

Now, the explicit formulas for the expected value and for the variance function of a thin rectangular plate response due to a two opposite train of random forces (vehicles) were derived. In order to have a complete investigation of the dynamic behaviors of the studied system, the numerical analysis will be carried out in which the effects of some main parameters of the moving loads on the probabilistic bridge response will be analysed in the following section.

\subsection{Numerical results and discussion}

For the numerical purpose, the case of bridge plate having the following parameters are considered (see Table 1). It is assumed that the vehicles weight had a lognormal distribution with the expected value and the standard deviation equal to $\mathrm{E}[A]=10^{5} \mathrm{~N}$ and $\mathrm{E}\left[A^{2}\right]=1.2 \times 10^{10} \mathrm{~N}^{2}([16])$, respectively. In this paper the problem will be confined to the homogeneous case. Therefore, the intensity of the load distribution is assumed to be constant for the simplicity: $\lambda_{j}(\tau)=\lambda=0.33 / \omega_{0}$ ([14]). Nevertheless, notice that, in traffic engineering [15], this intensity is related to the average passage speed of the vehicles and inversely. The mean value of the velocity $v_{o j}$ varying from 10 to $50 \mathrm{~m} / \mathrm{s}$; the variation coefficient of velocity $\sigma_{v j}$ varying from 0.01 to 0.3 . We assume that $u_{01}=u_{02}=u_{0}$ and $\sigma_{v 1}=\sigma_{v 2}=\sigma_{v}$. The

Table 1: Properties of the model of plate studied ([7])

\begin{tabular}{c|c|c}
\hline Item & Notation & Value \\
\hline Length & $a$ & $24 \mathrm{~m}$ \\
\hline Width & $b$ & $8 \mathrm{~m}$ \\
\hline Thickness & $h$ & $1 \mathrm{~m}$ \\
\hline Young's modulus & $E$ & $50 \mathrm{GPa}$ \\
\hline Poisson's ratio & $v$ & 0.3 \\
\hline Mass density & $\rho$ & $2400 \mathrm{~kg} / \mathrm{m}^{3}$ \\
\hline damping coefficient & $c$ & $0.91 \mathrm{~kg} / \mathrm{s}$ \\
\hline
\end{tabular}

presents numerical analysis is carried out by simulating first the deterministic Eqs. ((3.3) and (3.5)) through the fourth order Runge-Kutta algorithm. Therefore, the expected $\mathrm{E}[w(x, y, t)]$ and the standard deviation $\sigma_{w}^{2}(x, y, t)$ of the dimensionless plate displacement at the midspan $(x=1 / 2, y=1 / 2)$ are obtained for different range of the moving loads parameters.

In Fig. 3.1, the average plate deformation is shown when various numbers of modes are used to analyze the response of the structure. It is quite evident that moving from a single mode (mode $(1,1)$ ) analysis to another one, mode $(1,2)$ the accuracy of the solution drastically 
(a)

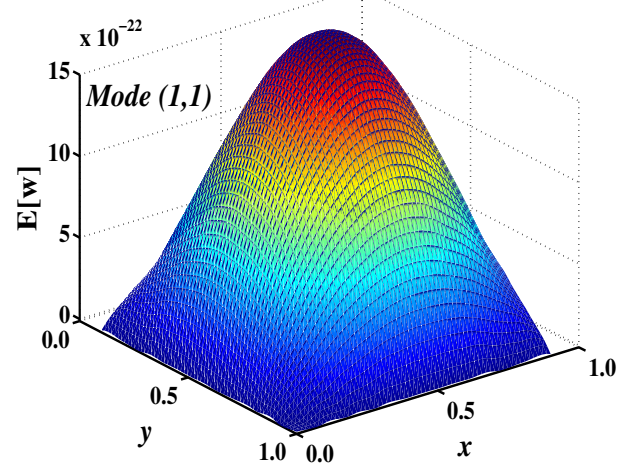

(c)

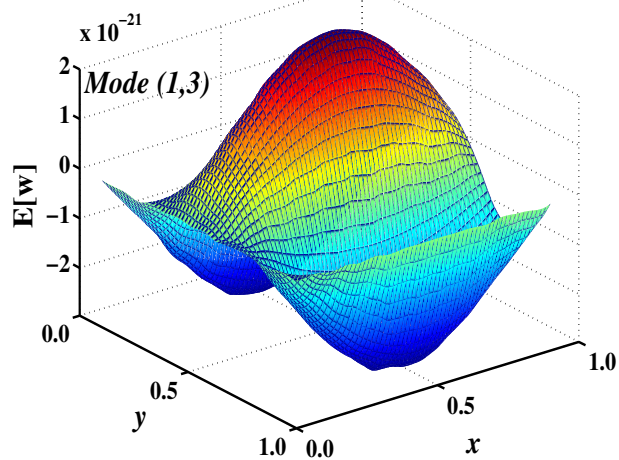

(b)

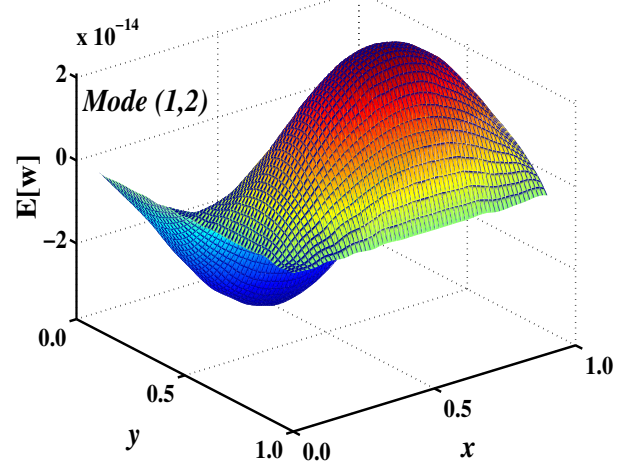

(d)

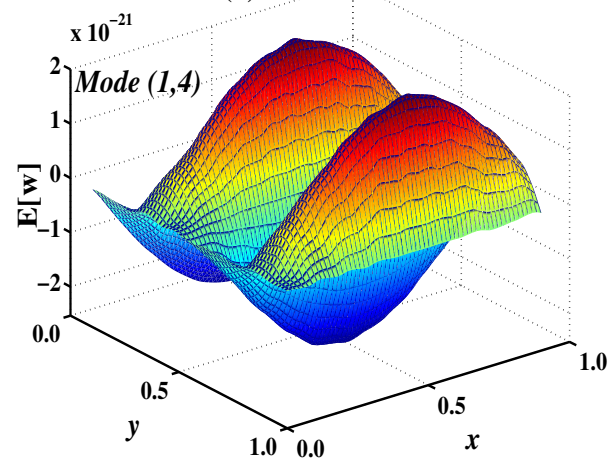

Figure 3.1: Average plate deformation when $\lambda_{1}=\lambda_{2}=0.15, \sigma_{v 1}=\sigma_{v 2}=0.3, u_{01}=u_{02}=0.5$. (a) mode $(1,1)$, (b) mode $(1,2)$, (c) mode (1,3) and (d) mode $(1,4)$. The parameters used are obtained according to Eqs. $((2.7),(2.14))$ and Table 1
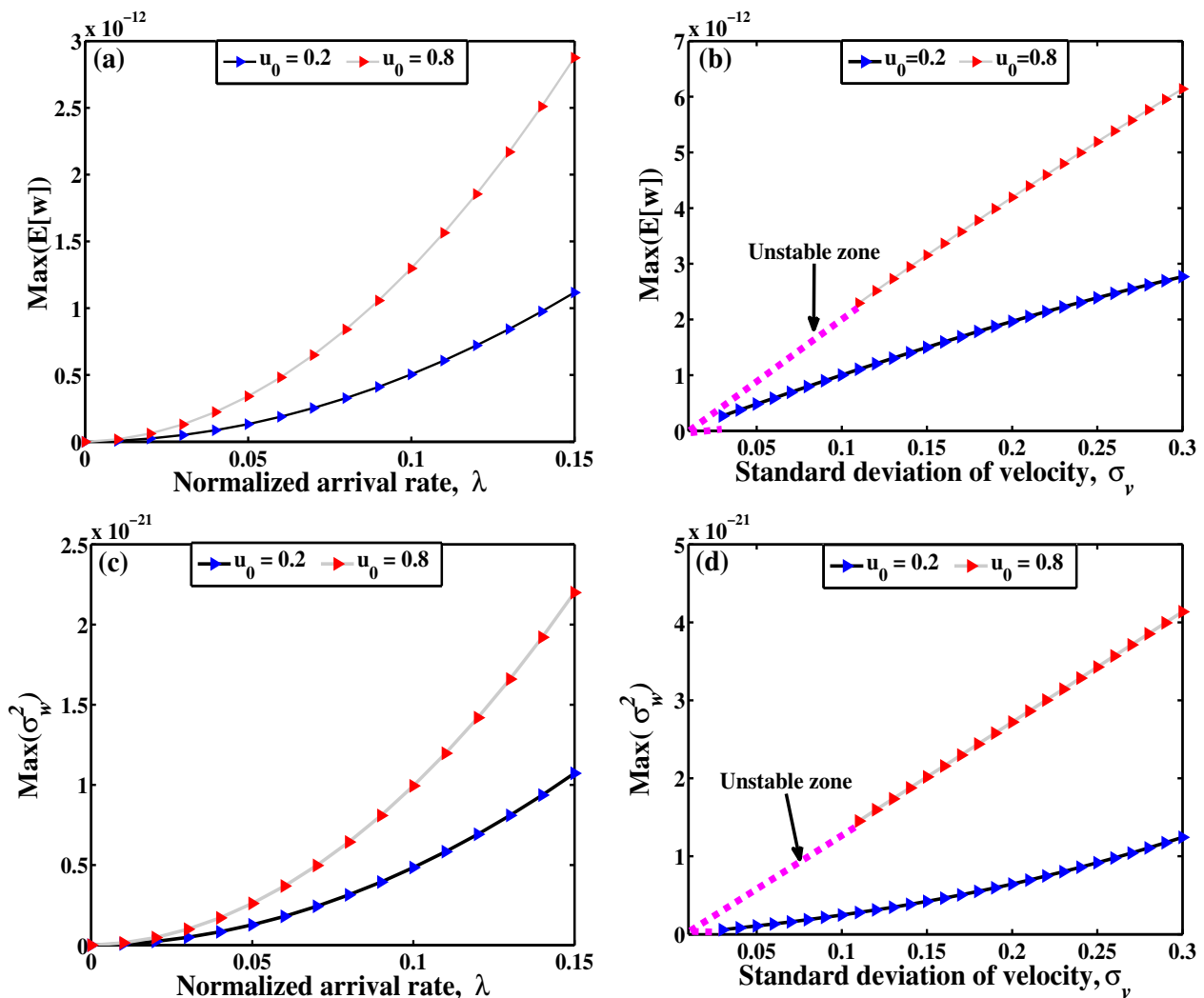

Figure 3.2: The maximum expected values and the variances of a plate displacement in relation to the normalized average flow intensity $\lambda$ or the standard deviation of the stochastic velocity $\sigma_{v}$. (a), (b) expected value, (c), (d) variance. The other parameters used are obtained according to Eqs. ((2.7), (2.14)) and Table 1. 
increases and an abrupt change in the shape of the plate is observed. This can be explain by the fact that, moving load is commonly expected to excite more modal shape, not only the first mode. Likewise, increasing the number of modes in the analysis to $(1,4)$ apparently decreases the solution.

Fig. 3.2 illustrates the relations between the normalized average flow intensity $\lambda$ or the standard deviation of the stochastic velocity and the expected values and variances of a stochastic maximal plate displacement $w(x, y, t)$. It is observed first that as the value of $\lambda$ increases the expected $\mathrm{E}[w]$ and standard deviation $\sigma_{w}^{2}$ of a maximal plate deflection merely increase (see Figs. 3.2(a) and 3.2(c)). Since that the number of vehicles $N(t)$ is related to the arrival rate $\lambda$ and inversely, this result confirms well the one obtains by [7] and by [17]. Secondly, the increase of the value of $\sigma_{v}$ also increases the expected and the standard deviation of a maximal plate deflection (see Figs. 3.2(b) and 3.2(d)). The analysis leads us to the conclusion that it is necessary and more important to take into account the stochastic nature of the load velocity in such studies. The influence of the mean velocity on the same probabilistic features of the plate response is also investigated and it is observed that as the mean value of the velocities increases, the expected value and the variance of the plate response also increase. It is also observed that for a normalized average speed $u_{0}=0.8($ about $50 \mathrm{~m} / \mathrm{s})$, an unstable zone is observed when the standard deviation of the velocity $\sigma_{v} \in[0.01,0.11]$. This unstable zone decreases as the mean value velocity decreases.

\section{Summary and conclusion}

Summing up, an approach to analyse the stochastic vibrations of a two lane slab-type-bridge due to a set of random point forces, which idealise the traffic flow, moving with stochastic velocities has been presented. This approach has been built under the assumption that the force arrival times constitute a Poisson stochastic process and the velocity of each force is a special kind of stochastic process, which can be described by the sum of the mean value and a white noise disturbance. By using the Kirchhoff's hypothesis, a two lane slab-type-bridge has been modelled as a Simply Supported thin rectangular plate with two separate rectilinear paths. The results prove the strong influence of the variation coefficient of velocity on the plate response - both the expected value and the standard deviation of deflection. It has been observed that the bridge safety strongly depends to the mean value and to the standard deviation of the vehicles velocities. Thus, some bridges may last more than expected or prematurely destroy because of the effects of the moving load random velocities.

On the basis of the approach presented above the influence of different traffic load models parameters on the random response of a bridge can be studied. It enables us to select the optimum design solution from a reliability point of view.

\section{References}

[1] L. Frýba, Vibration of Solids and Structures under Moving Load, Thomas Telford, London, 1999.

[2] H. Ouyang, Moving load dynamic problems: a tutorial (with a brief overview), Mechanical Systems and Signal Processing, 25 (2011), $2039-2060$.

[3] A. Nikkhoo, F. R. Rofooei, Parametric study of the dynamic response of thin rectangular plates traversed by a moving mass, Acta Mechanica, 223 (2012), 15-27.

[4] J.-J. Wu, Vibration analyses of an inclined flat plate subjected to moving loads, Journal of Sound and Vibration, 299 (2007), $373-387$.

[5] J. Vaseghi Amiri, A. Nikkhoo, M. R. Davoodi, M. Ebrahimzadeh Hassanabadi, Vibration analysis of a Mindlin elastic plate under a moving mass excitation by eigenfunction expansion method, Thin-Walled Structures, 62 (2012), 53-64.

[6] X. Q. Zhu, S. S. Law, Dynamic Behavior of Orthotropic Rectangular Plates under Moving Loads, Journal of Engineering Mechanics, 129 (2007), 79-87.

[7] A. Nikkhoo, M. Ebrahimzadeh Hassanabadi, S. Eftekhar Azamc, J. Vaseghi Amiri, Vibration of a thin rectangular plate subjected to series of moving inertial loads, Mechanics Research Communications, 55 (2014), 105-113.

[8] A. Rystwej, P. Śniady, Dynamic response of an infinite beam and plate to a stochastic train of moving forces, Journal of Sound and Vibration, 299 (2007), 1033-1048.

[9] M. Li, T. Qian, Y. Zhong, H. Zhong, Dynamic Response of the Rectangular Plate Subjected to Moving Loads with Variable Velocity, Journal of Engineering Mechanics, 140 (2013), 06014001.

[10] S. P. Timoshenko, Theory of Plates and Shells, Wiley, New York, 1959.

[11] P. Śniady, S. Biernat, R. Sieniawska, S. Zukowski, Vibrations of the beam due to a load moving with stochastic velocity, Probabilistic Engineering Mechanics, 16 (2001), 53-59.

[12] L. M. Anague Tabejieu, B. R. Nana Nbendjo, G. Filatrella, P. Woafo, Amplitude stochastic response of Rayleigh beams to randomly moving loads, Nonlinear Dynamics, 89 (2017), 925-937.

[13] W.-C. Xie, Dynamic stability of structures, Cambridge university press, New York, 2006.

[14] P. Śniady, S. Biernat, R. Sieniawska, S. Zukowski, Vibrations of the beam due to a load moving with stochastic velocity, Probabilistic Engineering Mechanics, 16 (2001), 53-59.

[15] R. J. Salter, Highway Trafic Analysis and Design, Macmillan, London, 1976.

[16] D. Bryja, P. Śniady, Spatially coupled vibrations of a suspension bridge under random Highway traffic, Earthquake Engineering and Structural Dynamics, 20 (1991), 995-1010.

[17] L. M. Anague Tabejieu, B. R. Nana Nbendjo, U. Dorka, Identification of horseshoes chaos in a cable-stayed bridge subjected to randomly moving loads, International Journal of Non-Linear Mechanics, 85 (2016), 62-69.

[18] M. Kamiński, The Stochastic Perturbation Method for Computational Mechanics, John Wiley \& Sons Ltd, United Kingdom, 2013. 